Research Paper

\title{
IFN-DC Loaded with Autophagosomes containing Virus Antigen is Highly Efficient in Inducing Virus-Specific Human T Cells
}

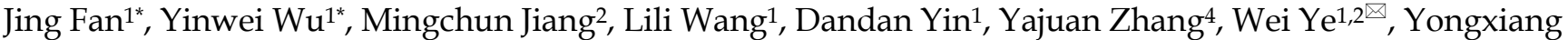 \\ $\mathrm{Yi}^{1}, 3$ \\ 1. Clinical Research Center, The Second Hospital of Nanjing, Nanjing University of Chinese Medicine. Zhong Fu Road, Gulou District, Nanjing, Jiangsu, PR \\ China 210003. \\ 2. Out-patient department, Nanjing Army Command College, Nanjing, 210045, China. \\ 3. Liver Disease, The Second Hospital of Nanjing, Nanjing University of Chinese Medicine. Zhong Fu Road, Gulou District, Nanjing, Jiangsu, PR China 210003. \\ 4. Department of Hepatobiliary Surgery, The Second Hospital of Nanjing, Nanjing University of Chinese Medicine. Zhong Fu Road, Gulou District, Nanjing, \\ Jiangsu, PR China 210003. \\ 5. Health Management Center, Danyang People`s Hospital, Zhenjiang, 212300, China. \\ *These authors contributed equally to this work
}

$\triangle$ Corresponding authors: Wei Ye, email: yeweiseu@163.com; phone: +8613951729085; address: Clinical Research Center, The Second Hospital of Nanjing, Nanjing University of Chinese Medicine. Zhong Fu Road, Gulou District, Nanjing, Jiangsu, PR China 210003.

(c) Ivyspring International Publisher. This is an open access article distributed under the terms of the Creative Commons Attribution (CC BY-NC) license (https:// creativecommons.org/licenses/by-nc/4.0/). See http://ivyspring.com/terms for full terms and conditions.

Received: 2018.11.27; Accepted: 2019.03.27; Published: 2019.05.10

\begin{abstract}
Autophagy plays a critical role in the regulation of innate and adaptive immune responses to pathogens and tumors. A previous study utilized proteasome and lysosome inhibitors to form autophagosomes (DRibbles) and the effect of dendritic cells (DCs) loaded with DRibbles in activating antigen-specific T cells has been demonstrated in a mouse experiment and human IL-4-DC. In this study, CMV-DRibbles derived from MDA cell lines expressing cytomegalovirus (CMV) Pp65 protein were loaded onto human IFN-DC and IL-4-DC derived from monocytes, respectively. We observed that CMV-DRibbles resulted in the up-regulation of HLA-DR, CDIlc, and CD83, but not co-stimulatory molecules CD 80 and CD86 on IFN-DC. Meanwhile, the expression of HLA-DR, CD80, CD83, and CD86, except for CD11c on IL-4-DC loaded with CMV-DRibbles were up-regulated. Moreover, CMV-DRibbles had no ability to stimulate these two moDCs to secrete cytokines IL-6, IL-1 $\beta$ and IL-10. Then, we optimized the conditions for antigen up-take by DCs and found that mature moDCs had a superior ability to up-take CMV-DRibbles compared with immature DCs in a dose-dependent manner. Furthermore, the efficiency of CMV-DRibbles up-take by IFN-DC was superior compared to IL-4-DC. Finally, we observed that mIFN-DC was significantly more efficient at stimulating autologous CMV-specific CD4+ $T$ cells $(0.39$ vs. $0.28 \%, p<0.05)$ and $C D 8+T$ cells $(0.36$ vs. $0.12 \%, p<0.05)$ to secrete IFN-Y compared with mIL-4-DC. Therefore, DRibbles containing specific viral antigens were efficient activators of human antigen-specific $T$ cells. Our results demonstrated that IFN-DC loaded with CMV-DRibbles revealed a superior ability to induce CMV-specific T cells.
\end{abstract}

Key words: autophagosome, cancer vaccine, cross-presentation, dendritic cells

\section{Introduction}

Dendritic cells (DCs), as the most potent professional antigen-presenting cells (APCs), play a significant role in the activation of antiviral responses, autoimmune disease, maintenance in inflammatory skin orders, and anti-cancer responses. Due to the low frequency of DCs $(<1 \%)$ in human peripheral blood mononuclear cells (PBMCs), most studies use monocyte-derived DCs (moDCs) as APCs in vitro[1]. Traditionally, researchers use granulocyte-macrophage colony-stimulating factor (GM -CSF) and interleukin (IL)-4 to stimulate monocytes to differentiate into IL-4-DC. A series of clinical trials have used 
IL-4-DC as APCs, and achieved certain effects[2]. For example, Michal et al. [3] verified that the combined chemoimmunotherapy with IL-4-DC and docetaxel was safe and resulted in longer than expected survival in patients with metastatic, castration-resistant prostate cancer. IL-4-DC vaccines have also proved to prolong survival in patients with metastatic melanoma[4]. In recent years, it has been reported that interferon (IFN)- $\alpha$ has the functions of antiviral, antitumor, and immunoregulatory activities. Evidence in the literature suggests that monocytes can differentiate into moDCs in the presence of GM-CSF and IFN- $\alpha$ and are referred to as IFN-DC[5]. However, the difference in efficiency of antigens presentation between IFN-DC and IL-4-DC has not been explored yet.

Autophagy is a fundamental catabolic pathway in which unnecessary or dysfunctional proteins and organelles are sequestered in double-membrane autophagosomes and delivered to lysosomes for degradation and recycling[6]. Increasing evidence suggests autophagy plays an important role in both innate and adaptive immunity $[7,8]$. It has been reported that autophagy not only participates in major histocompatibility complex class I (MHC I) presentation but also contributes to major histocompatibility complex class II (MHC II) presentation[9]. Previous studies suggest that autophagy in tumor cells plays a crucial role in cross-presentation and identifies autophagosomes as the efficient carriers for tumor-associated antigens (TAA) $[10,11]$. When treating tumor cells with inhibitors of proteasomes and lysosomes, a broad spectrum of cellular antigens, including long-lived proteins, ubiquitinated short- lived proteins (SLiPs), and defective ribosomal products (DRiPs), can be sequestered in autophagosomes. These autophagosomes are designated as DRibbles (defective ribosomal products in blebs). It has been proven that moDCs could present DRibbles and activate antigen-specific $T$ cells in murine models[12]. Ye et al have proven that IL-4-DC could present DRibbles to human antigenspecific $T$ cells[13]. However, the different in the ability of IL-4-DC and IFN-DC, from human PBMCs in vitro, present $\mathrm{DRibbles}$ and activate antigen-specific $\mathrm{CD}^{+}$and $\mathrm{CD}^{+} \mathrm{T}$ cells has not yet been determined.

In this study, CMV-DRibbles were initially extracted from MDA cell lines generated from breast cancer containing CMV pp65 protein. Then, whether CMV-DRibbles could affect the phenotypes of IFN-DC and IL-4-DC was explored. Next, the influence of CMV-DRibbles on cytokines secretion of moDCs was determined. Moreover, the optimal conditions of DRibbles uptake by these two moDCs were assessed. At last, the comparison of the ability of presenting CMV-DRibbles between IFN-DC and
IL-4-DC were explored.

\section{Materials and Methods}

\section{Human blood donors and preparation of PBMCs}

Heparinized peripheral blood samples were obtained from healthy volunteers. PBMCs were Isolated using Ficoll density gradient centrifugation (TBD, Tianjin, China) and cultured in X-vivo ${ }^{\mathrm{TM}} 15$ medium (Lonza Group, Basel, Switzerland) containing $5 \%$ human AB serum, 100 units $/ \mathrm{ml}$ penicillin, and 100 $\mu \mathrm{g} / \mathrm{ml}$ streptomycin. The study was approved by the medical ethical committee of The Second Hospital of Nanjing, and written informed consents were obtained from all donors in accordance with the Declaration of Helsinki (1964) before blood collection.

\section{Cell separation and DCs generation}

Purified monocytes were isolated by anti-CD14 ${ }^{+}$ magnetically labeled microbeads (MiltenyiBiotec, Bergisch Gladbach, Germany). Positively selected $\mathrm{CD} 14^{+}$cells were analyzed by flow cytometry, and the purity was $>98 \%$. Purified monocytes were cultured in X-vivo ${ }^{\mathrm{TM}} 15$ medium containing $5 \%$ human $\mathrm{AB}$ serum, 100 units $/ \mathrm{ml}$ penicillin, and $100 \mu \mathrm{g} / \mathrm{ml}$ streptomycin at the concentration of $1 \times 10^{6} / \mathrm{ml}$, supplemented with $1000 \mathrm{U} / \mathrm{ml}$ IFN-a2b (MiltenyiBiotec, Bergisch Gladbach, Germany) and $40 \mathrm{ng} / \mathrm{ml}$ GM-CSF (R\&D Systems, Minneapolis, MN, USA) for IFN-DC, or $20 \mathrm{ng} / \mathrm{ml}$ IL-4 (R\&D Systems, Minneapolis, MN, USA) and $40 \mathrm{ng} / \mathrm{ml}$ GM-CSF for IL-4-DC. Immature IFN-DC (imIFN-DC) were incubated at 37 ${ }^{\circ} \mathrm{C}$ and $5 \% \mathrm{CO}_{2}$ for 3 days, and immature IL-4-DC (imIL-4-DC) were incubated for 5 days. Half of the supernatants were removed, and fresh cytokines and medium were added every 3 days. DCs were matured by adding $20 \mathrm{ng} / \mathrm{ml}$ TNF-a (R\&D Systems, Minneapolis, MN, USA) and cultured for another $48 \mathrm{~h}$.

\section{Tumor cell lines and preparation of DRibbles}

MDA human breast cancer cells or MDA human breast cancer cells expressing the CMV pp65 protein, were cultured in RPMI1640 medium containing $10 \%$ FBS, 100 units/ml penicillin, and $100 \mu \mathrm{g} / \mathrm{ml}$ streptomycin. Autophagosomes-enriched DRibbles were prepared as described previously[11]. Briefly, cells were treated with $10 \mathrm{mmol} / \mathrm{L} \mathrm{NH}_{4} \mathrm{Cl}$ and $100 \mathrm{nmol} / \mathrm{L}$ Bortezomib (Velcade) for 48 hours. After digestion by pancreatin, cells were pelleted by centrifugation at $300 \times g$ for 5 minutes three times. The resulting suspension was centrifuged at $7500 \times g$ for 10 minutes to collect the DRibbles. The MDA-DRibbles were extracted from MDA human breast cancer cells, as the CMV-DRibbles were extracted from MDA human breast cancer cells expressing the CMV-pp65 protein. 
The concentration of DRibbles was measured by a BCA protein assay kit (Thermo Fisher Scientific, Rockford IL, USA) according to the manufacturer's protocols.

\section{CFSE-labeling and assay for phagocytic ability}

MDA human breast cancer cells expressing the CMV-pp65 protein were labeled by CFSE (Invitrogen, Carlsbad, CA). The final concentration of CFSE was 5 $\mu \mathrm{M}$. The CMV-DRibbles were extracted as described above. Immature or mature DCs $\left(0.5 \times 10^{6} / \mathrm{mL}\right)$ were incubated at $37{ }^{\circ} \mathrm{C}$ with different concentrations of DRibbles for different time if necessary. After being washed twice, cells were resuspended for detection by a FACS Canto II flow cytometer (BD Biosciences, San Jose, CA, USA). DCs incubated at $4{ }^{\circ} \mathrm{C}$ with DRibbles were used as a negative control.

\section{Flow cytometric analysis}

DCs were incubated with or without DRibbles for 24 hours at $37^{\circ} \mathrm{C}$. Then, the cells were washed and resuspended in PBS containing $1 \%$ FBS and incubated with a series of monoclonal antibodies (mAbs) including anti-HLA-DR (G46-6), CD11c (B-ly6), CD14 (M5E2), CD80 (16-10A1), CD83 (Michel19), and CD86 (GL1) (BD PharMingen, San Diego, CA, USA) for 30 minutes at $4{ }^{\circ} \mathrm{C}$. Anti-HLA-DR mAb conjugated with PerCp, anti-CD11c mAb conjugated with PE-CY7, anti-CD14 mAb conjugated with FITC, anti-CD80 mAb conjugated with PE, anti-CD83 mAb conjugated with $\mathrm{PE}$, and anti-CD86 $\mathrm{mAb}$ conjugated with PE. Then, the samples were analyzed by a FACS Canto II flow cytometer (BD Biosciences, San Jose, CA, USA). Data were collected with BD FACSDiva software and analyzed with Treestar Flowjo software.

\section{Cytokines secretion analysis}

The cytokines secretion of DCs loaded with CMV-DRibbles were analyzed by standard sandwich ELISA. Supernatants were analyzed for IL-6, IL-1 $\beta$, and IL-10 according to the manufacturer's protocol (Multi sciences, Hangzhou, China).

\section{Western blot analysis}

MDA cells with or without the expression of CMV pp65 protein were treated with $10 \mathrm{mmol} / \mathrm{L}$ $\mathrm{NH}_{4} \mathrm{Cl}$ and $100 \mathrm{nmol} / \mathrm{L}$ Bortezomib. Meanwhile, the untreated MDA cells served as control. The procedures of DRibbles extraction were described in section 2.3. Then, DRibbles were mixed with $5 \times$ SDS sample loading buffer and samples were resolved by $4-12 \%$ SDS-PAGE. Proteins were transferred to polyvinylidene fluoride membranes, incubated with blocking buffer for 2 hours, incubated with primary antibody overnight and with HRP-conjugated secondary antibodies for 2 hours. Protein bands were revealed by using chemiluminescent. The primary antibodies included anti-CMV-pp65 antibody (1:1000, Santa Cruz, Dallas, USA), anti-LC3 antibody (1:1000, Abcam, Cambridge, UK), and anti-Tubulin antibody (1:3000, CMCTAG, Milwaukee, USA). The secondary antibodies were goat-anti-mouse-HRP (1:10000, Thermo Fisher Scientific, Rockford IL, USA) and goat-anti-rabbit-HRP $\quad(1: 10000, \quad$ Thermo Fisher Scientific, Rockford IL, USA).

\section{Analysis of antigen-specific $T$ cells by intracellular IFN-Y staining}

At first, the healthy donors with positive anti-CMV-IgG were selected to do the study according to the results of the ELISA (Neobioscience, Shenzhen, China). DCs cultured as above were seeded into a 96-well round-bottomed plate at $5 \times 10^{4}$ cells per well. Then, mature IFN-DC (mIFN-DC) or matue IL-4-DC (mIL-4-DC) was cultured with CMVDRibbles. Purified $\mathrm{CD}^{+}$or $\mathrm{CD}^{+} \mathrm{T}$ cells were isolated by anti-CD4 or anti-CD8 magnetically labeled microbeads according to the manufacturer`s protocols (MiltenyiBiotec, Bergisch Gladbach, Germany). After 12 hours, DCs loaded with CMV-DRibbles were washed twice in PBS and co-cultured with autologous $\mathrm{CD}^{+}$or $\mathrm{CD}^{+} \mathrm{T}$ cells at the DCs/T cells ratio of 1:10 in $\mathrm{X}$-vivo ${ }^{\mathrm{TM}} 15$ containing $5 \%$ human $\mathrm{AB}$ serum, 100 units $/ \mathrm{ml}$ penicillin, and $100 \mu \mathrm{g} / \mathrm{ml}$ streptomycin for 12 hours at $37^{\circ} \mathrm{C}$. Then, GolgiPlug protein transport inhibitor (BD PharMingen, San Diego, CA, USA) was added into the wells. After another 6 hours, cells were harvested and washed in washing buffer and stained with live/dead fixable dead cell staining (Invitrogen, Carlsbad, CA), FITC-conjugated anti-CD4, APC-CY7conjugated anti-CD8 and PerCp-conjugated anti-CD3 (BD PharMingen, San Diego, CA, USA) for 30 minutes at $4{ }^{\circ} \mathrm{C}$. After washing, the cells were fixed and permeabilized by the Cytofix/Cytoperm solution (BD PharMingen, San Diego, CA, USA) for 20 minutes at 4 ${ }^{\circ} \mathrm{C}$. Then the cells were rewashed in perm washing buffer and stained with PE-conjugated anti-IFN-Y (BD PharMingen, San Diego, CA, USA) for 30 minutes at 4 ${ }^{\circ} \mathrm{C}$. At last, the cells were analyzed on a BD Canto II flow cytometer.

\section{Statistical Analysis}

Data were expressed as the means \pm SEMs and analyzed by Student's $t$-test or a one-way ANOVA followed by Tukey testing using SPSS V20.0 software. Statistical significance was defined as $<0.05$.

\section{Results}

DRibbles induced the modification of phenotypes on moDCs

A previous study had shown that DRibbles 
could change the phenotype of IL-4-DC derived from mouse bone marrow mononuclear cells[12]. Therefore, we wondered if DRibbles could modulate the phenotypes of DCs derived from human PBMCs. Isolated human monocytes were cultured either with GM-CSF plus IL-4 (IL-4-DC) or GM-CSF plus IFN-a (IFN-DC). After cultured for 5 days for IL-4-DC and 3 days for IFN-DC, the cells were added with CMV-DRibbles and cultured for another 24 hours. Flow cytometry was used to analyze co-stimulation markers (HLA-DR, C80, CD86), myeloid marker (CD11c), and DC mature marker (CD83). After the stimulation of CMV-DRibbles, the expression of MHC II molecules HLA-DR and myeloid markers CD11c and DC mature marker CD83 on imIFN-DC were upregulated $(p<0.05)$, analyzed as the percentage of positive cells as well as by MFI (Fig. 1A and B). Moreover, the expression of costimulatory molecules CD80 and CD86 before and after the stimulation CMV-DRibbles on imIFN-DC had no significant difference $(p>0.05)$. For IL-4-DC, the expression of CD11c could not be increased remarkably by the stimulation of CMV-DRibbles $(p>0.05)$, whereas the expression of HLA-DR, CD80, CD83, and CD86 could be upregulated $(p<0.05)$ after loading with CMV-DRibbles. However, except for CD86, there was no significant difference $(p>0.05)$ for the expression of HLA-DR, CD11c, CD80, and CD83 between IFN-DC and IL-4-DC after the stimulation of CMV-DRibbles.

\section{Effect of DRibbles on the cytokines secretion of two moDCs}

As DRibbles were able to efficiently influence the phenotypes of moDCs, we tested whether DRibbles could stimulate cytokines secretion by moDCs. To evaluate the effect of DRibbles on the regulation of cytokines secretion by IFN-DC and IL-4-DC in vitro, the levels of cytokines in cell culture supernatants after the stimulation of CMV-DRibbles were detected by ELISA. ImIFN-DC and imIL-4-DC were generated and maturated for 48 hours with TNF-a. Afterwards, mature DCs were loaded with CMV-DRibbles and further cultured for 24 hours. Levels of IL-6, IL-1 $\beta$, and IL-10 in supernatants were determined. In comparison to mIL-4-DC, mIFN-DC secreted more IL-6 (Fig. 2A). However, CMV-DRibbles had no ability to further enhance the concentration of IL-6 secreted by mIFN-DC and mIL-4-DC $(p>0.05)$. Moreover, imIFN-DC and imIL-4-DC did not secrete detectable levels of IL-1 $\beta$ (Fig. 2B). After maturation by TNF- $\alpha$, both mIFN-DC and mIL-4-DC released significant amounts of IL-1 $\beta$, and the concentration of IL-1 $\beta$ secreted by mIFN-DC was lower than mIL-4-DC $(p<0.001)$. Nevertheless, the concentration of IL-1 $\beta$ released by mIFN-DC and mIL-4-DC was decreased after loaded with CMV-DRibbles $(p<0.001)$. Surprisingly, all of imIL-4-DC, mIL-4-DC and mIL-4-DC loaded with CMV-DRibbles did not secrete detectable levels of IL-10 (Fig. 2C). In contrast, imIFN-DC secreted IL-10. Although mIFN-DC produced higher levels of IL-10 compared with imIFN-DC, the secretion of IL-10 by mIFN-DC could not be further increased after loaded with CMV-DRibbles $(p>0.05)$.

\section{The uptake of DRibbles by IFN-DC and IL-4-DC}

To analyze the capacity of moDCs to uptake DRibbles, CMV-DRibbles were prepared from CFSE-labeled MDA cells expressing CMV pp65 protein. Then, the ability of uptake was compared between immature and mature moDCs. As shown in Fig $3 \mathrm{~A}$ and $\mathrm{B}$, both mIFN-DC and mIL-4-DC had higher capacity to uptake CMV-DRibbles compared with their corresponding immature DCs $(p<0.001)$. The negative control was cultured at $4{ }^{\circ} \mathrm{C}$. In addition, the ability of CMV-DRibbles uptake by IFN-DC was stronger than IL-4-DC no matter if they were immature or mature DCs $(p<0.001)$. Next, it was determined whether the concentration of CMVDRibbles could influence uptake efficiency. Dose analysis indicated that the uptake of CMV-DRibbles by mature DCs was in a dose-dependent manner (Fig. 3C). Finally, CMV-DRibbles uptake by mature DCs were analyzed at time points 6,12 , and 24 hours. The results showed that the best time point for the uptake of DRibbles by mIFN-DC was 12 hours; the best time for the mIL-4-DC was 24 hours (Fig. 3D).

\section{Comparison of the presentation ability between IFN-DC and IL-4-DC}

IFN-DC and IL-4-DC are the most commonly used APCs in immunotherapy. Currently, it is not known which DCs loaded DRibbles can more effectively activate antigen-specific T cells. Hence, we investigated the ability of IFN-DC and IL-4-DC to induce $\mathrm{T}$ cells secreting IFN- $\gamma$ after loading with CMV-DRibbles. CMV-DRibbles or MDA-DRibbles were extracted from MDA cells with or without the expression of CMV pp65 protein as shown in Fig. 4A. LC3-II was a typical marker of autophagosomes[14]. Western blot analysis showed that LC3-I to LC3-II conversion of DRibbles markedly increased after treatment with $\mathrm{NH}_{4} \mathrm{Cl}$ and Bortezomib (Fig. 4B). CMV IgG positive healthy donors' peripheral blood were selected for the next study. Cultured mIFN-DC or mIL-4-DC loaded with CMV-DRibbles or MDADRibbles were then co-cultured with autologous $\mathrm{CD}^{+}$and $\mathrm{CD}^{+} \mathrm{T}$ cells at a DC to $\mathrm{T}$ cells ratio of 1:10, $\mathrm{CD}^{+}$or $\mathrm{CD}^{+}{ }^{+} \mathrm{T}$ cells stimulated by $100 \mathrm{ng} / \mathrm{mL}$ PMA 
were used as positive control, and monocytes were used as a negative control. After 12 hours of culture, GolgiPlug protein transport inhibitor was added, and activated CMV-specific $\mathrm{T}$ cells were assessed by intracellular staining. Both mIFN-DC and mIL-4 DC had the capacity to prime $\mathrm{CMV}$-specific $\mathrm{CD}^{+}$and $\mathrm{CD}^{+} \mathrm{T}$ cells after loading with CMV-DRibbles when compared with loaded with MDA-DRibbles. As shown in Fig. 5A and 5B, mIFN-DC loaded with
CMV-DRibbles were more efficient at promoting $\mathrm{CMV}$ antigen-specific $\mathrm{CD}^{+} \mathrm{T}$ cells compared with the mIL-4-DC (0.39 \% vs. $0.28 \%, p<0.05)$. The mIFN-DC loaded with CMV-DRibbles could also activate more CMV antigen-specific $\mathrm{CD}^{+} \mathrm{T}$ cells than mIL-4-DC ( $0.36 \%$ vs. $0.12 \%, p<0.05)$ (Fig. $5 \mathrm{C}$ and $4 \mathrm{D}$ ). Taken together, these data suggested that human mIFN-DC had a stronger ability in the presentation of DRibbles antigen compared with mIL-4-DC.

A)
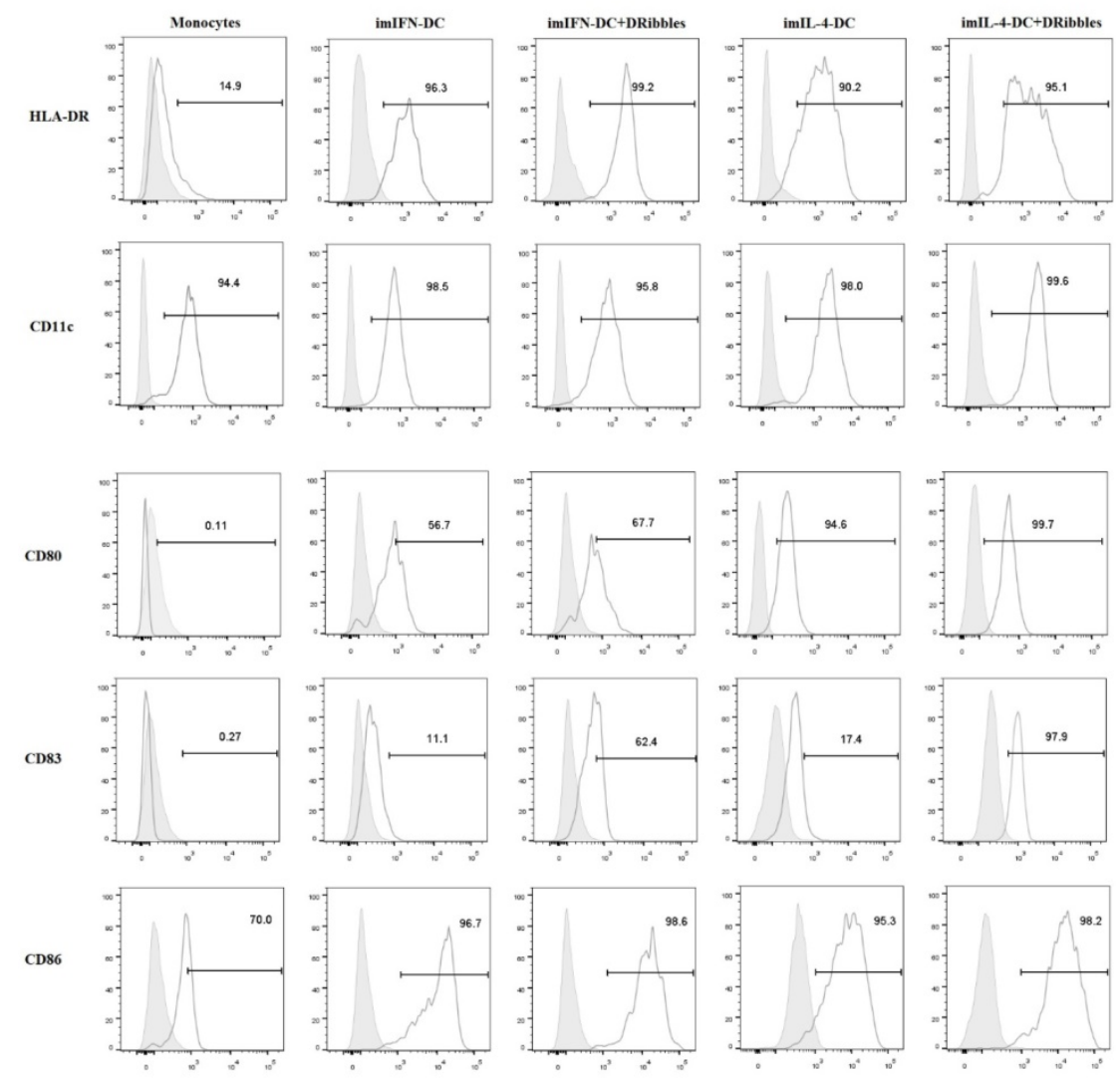

B)

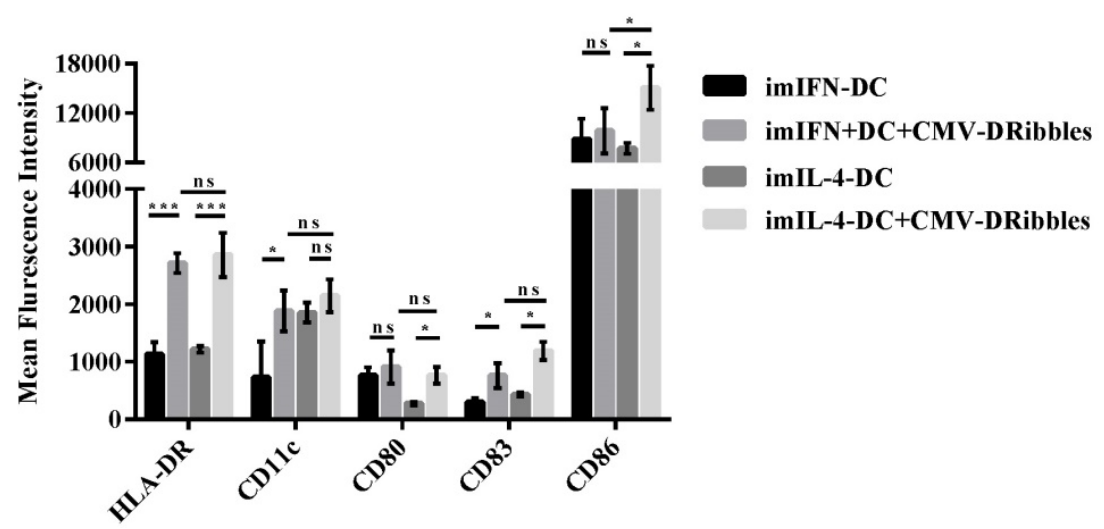

Figure 1. Phenotypes of imIFN-DC and imIL-4-DC before and after loading with CMV-DRibbles. (A) Phenotypic analysis of surface markers on monocytes, imIFN-DC, imIL-4-DC loaded with or without CMV-DRibbles. FACS analysis was performed on these cells that were cultured for 24 hours. Representative histograms (thick lines) of the indicated antibody staining were plotted with the corresponding isotype controls (shaded histograms). (B) showed the mean fluorescence intensity of HLA-DR, CD1 1c, CD80, CD83 and CD86 expressed by immature moDCs and immature moDCs loaded with CMV-DRibbles. Results were represented as means \pm SEMs obtained from 5 independent experiments from 5 different donors. Statistical analysis was performed with One-way ANOVA followed by post-hoc test $\left({ }^{*} p<0.05,{ }^{*} p<0.01, *^{* *} p<0.001\right.$, ns $=$ none sense). 
A)

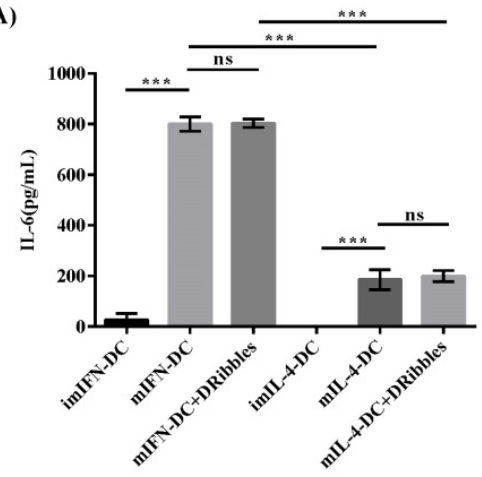

B)

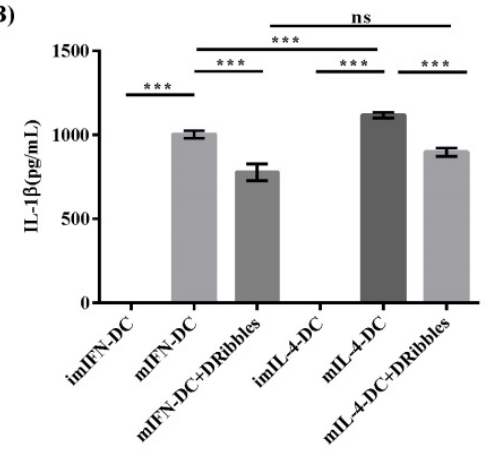

C)

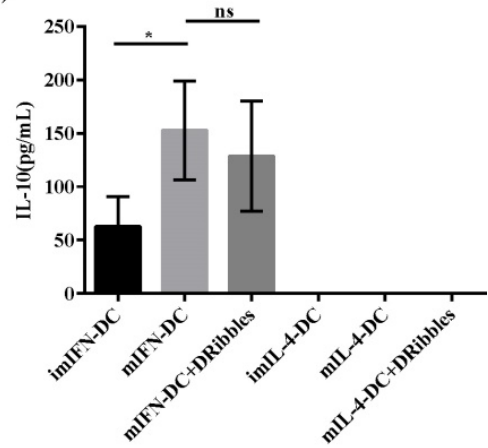

Figure 2. Cytokines produced by IFN-DC and IL-4-DC. (A), IL-6 (B), IL-1 $\beta$ (C), IL-10 secreted by immature moDCs, mature moDCs and mature moDCs loaded with CMV-DRibbles were analyzed by ELISA. Results were represented as means \pm SEMs obtained from 3 independent experiments from 3 different donors. Statistical analysis was performed with one-way ANOVA followed by post-hoc test $\left({ }^{*} p<0.05, * * p<0.01, * * * p<0.001\right.$, ns $=$ none sense) .

\section{Discussion}

In recent years, a number of cancer vaccines has been explored in the immunotherapy of different tumors, such as breast cancer, glioma, and liver cancer[15-17]. Most of these vaccines were comprised of peptide, protein, or nucleic acid, which could be captured by DCs in vitro or in vivo[18]. However, the clinical outcomes regarding these vaccines were unsatisfactory and the main reasons were as follows: (1) the weak immunogenicity of antigen when cross-presented by DCs; (2) the loss or defection of epitopes in antigen processing; (3) immune suppression induced by myeloid-derived suppressor cells and $\mathrm{T}$ regulatory cells. Therefore, appropriate tumor antigen and efficient cross-presentation by DCs are crucial factors in the process of developing tumor vaccines.

Autophagy is a fundamental catabolic pathway in which unnecessary or dysfunctional proteins and organelles are sequestered in double-membrane autophagosomes and delivered to lysosomes for the degradation and recycling during the maintenance of cellular homeostasis[6]. Some studies found that autophagosomes could be presented by the MHC II pathway. This process involves the degradation of antigens in lysosomal compartments, binding of the resulting peptides to MHC II, and delivery of the MHC II-peptide complexes to the cell surface for presentation to $\mathrm{CD}^{+} \mathrm{T}$ cells[19]. Autophagosomes can expand the repertoire of antigens presented via the MHC II pathway by DCs. Another route for presentation of exogenous antigens involved in autophagosomes is cross-presentation. This route involves the degradation of foreign antigens in the cytosol, binding of the resulting peptides to MHC I in the endoplasmic reticulum (ER), and delivery of the MHC I-peptide complexes to the plasma membrane for activation of $\mathrm{CD}^{+} \mathrm{T}$ cells[19]. DCs are the primary implementer of cross-presentation. Furthermore, DCs serve as the most potent APCs in human and is identified as the surveillance of extracellular environment, the capturer of pathogens, and the initiator of $\mathrm{T}$ cell immunity[20]. Consequently, developing a tumor vaccine based on autophagosomes and DCs is a promising way.

Previous studies used the inhibitors of proteasomes and lysosomes to process tumor cells and obtain autophagosomes containing quality antigens named DRibbles, which had been demonstrated to have value as a tumor vaccine in murine experiments[12]. DRibbles were induced by Bortezomib (proteasome inhibitor) and $\mathrm{NH}_{4} \mathrm{Cl}$ (lysosomotropic agent). Scanning electron microscopy analysis have shown that the dimensions and features of DRibbles are in accordance with autophagosomes[12, 21]. IL-4DC are conventional moDCs that are commonly used in clinical trials. In recent years, some reports suggest that monocytes cultured with GM-CSF and IFN-a can be induced toward the moDCs which are called IFN-DC[22]. Hence, in this study, we sought to determine whether IFN-DC and IL-4-DC derived from monocytes could present DRibbles to human T cells. For the first time, we demonstrated that DRibbles derived from the MDA cells line expressing CMV pp65 protein could change the phenotype of IFN-DC and IL-4-DC from human PBMCs. We also explored that IFN-DC loaded with CMV-DRibbles were more efficient in the activation of CMV antigen- specific $\mathrm{CD}^{+}$and $\mathrm{CD} 8^{+} \mathrm{T}$ cells compared with IL-4-DC. 
A)

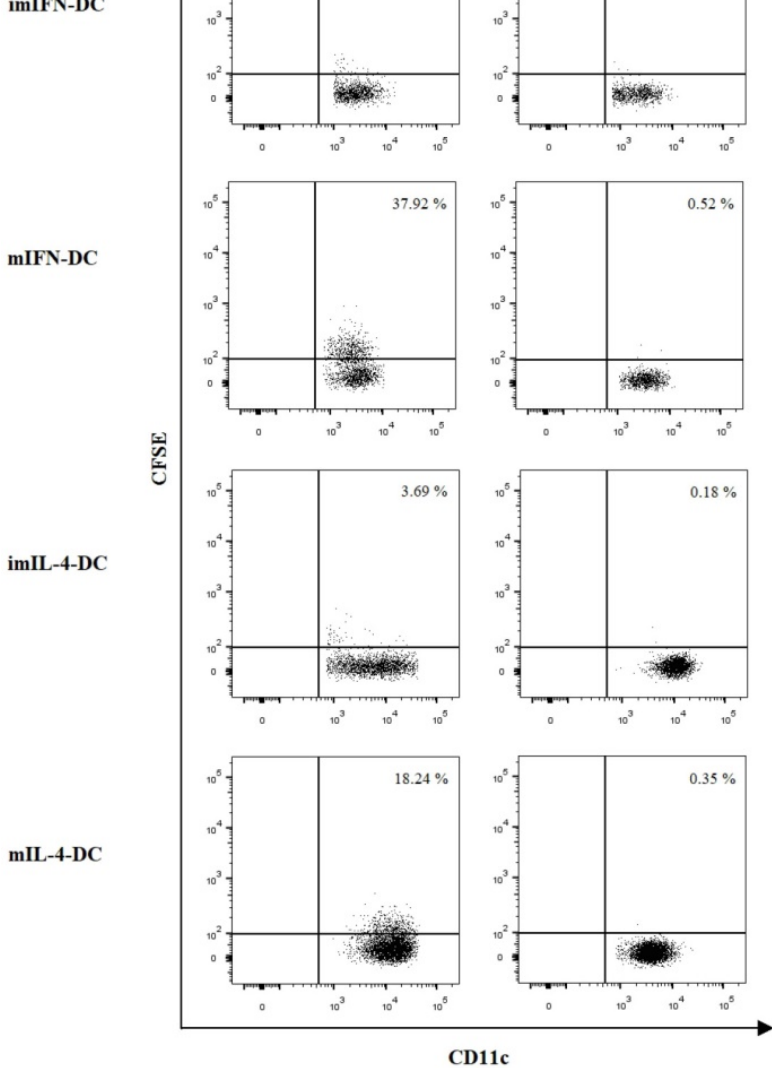

B)

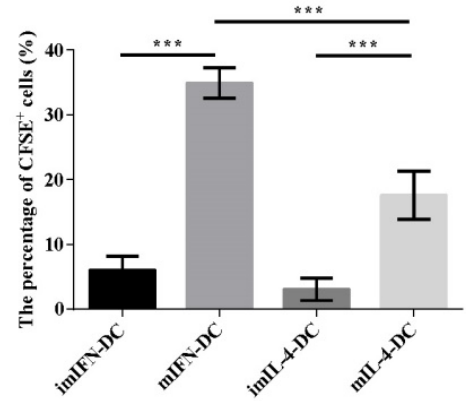

C)

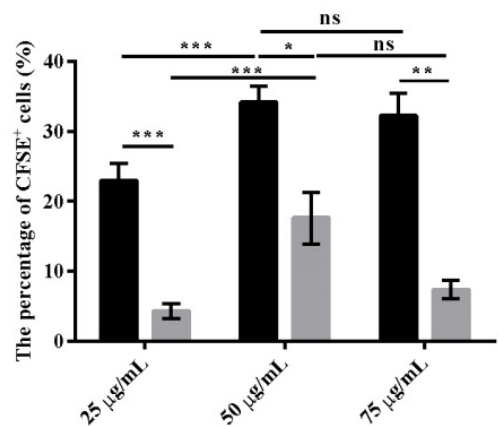

D)

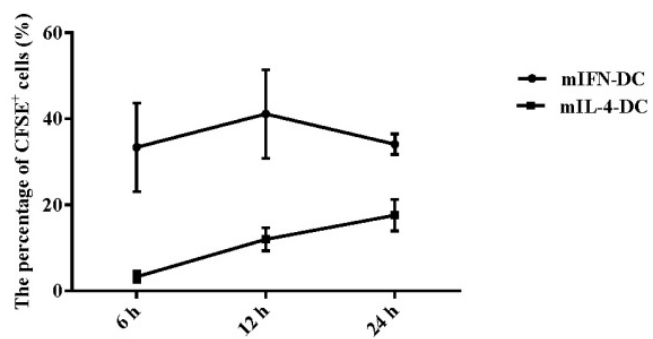

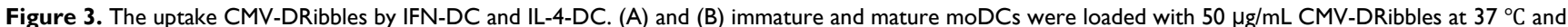
$4{ }^{\circ} \mathrm{C}$, respectively. After $24 \mathrm{~h}$, the cells were analyzed by flow cytometer. The percentage of CFSE ${ }^{+}$cells in the gate of CDIl c cells was determined. (C) mature DCs were loaded with $25 \mu \mathrm{g} / \mathrm{mL}, 50 \mu \mathrm{g} / \mathrm{mL}$ or $75 \mu \mathrm{g} / \mathrm{mL}$ CMV- DRibbles, respectively, and cultured at $37^{\circ} \mathrm{C}$ for $24 \mathrm{~h}$. Then, the percentage of CFSE ${ }^{+}$cells was detected. (D) mature moDCs were loaded with $50 \mu \mathrm{g} / \mathrm{mL}$ CMV-DRibbles for $6,12,24 \mathrm{~h}$ at $37^{\circ} \mathrm{C}$. Results were represented as means $\pm \mathrm{SEMs}$ obtained from 3 independent experiments. Statistical analysis was performed with One-way ANOVA followed by post-hoc test $(* p<0.05, * * p<0.01$, $* * * p<0.001$, ns $=$ none sense).

First, we detected a change of phenotype in the imIFN-DC and imIL-4-DC after loaded with CMV DRibbles. FACS analyses revealed that a large percentage of imIFN-DC and imIL-4-DC exhibited the typical characteristics of highly activated HLA-DR DCs after their exposure to CMV-DRibbles. This phenomenon was in concert with DCs cultured from mouse bone marrow mononuclear cells[12]. The primary function of HLA-DR is to present antigens to the immune system for eliciting $\mathrm{T}$ cells responses, and HLA-DR is a marker of immune stimulation[23]. Therefore, the increase of HLA-DR expression is beneficial for moDCs to stimulate T cells. For myeloid marker CD11c, the expression on imIFN-DC loaded with CMV-DRibbles increased, while the expression on imIL-4-DC increased slightly but there was no statistical change after the stimulation of CMV-DRibbles. The function of CD11c is to induce cellular activation, and CD11c is found on most dendritic cells, but also on monocytes, macrophages, neutrophils[24]. Hence, the upregulated expression of
CD11c by DRibbles is in favor of moDCs function. CD80 and CD86 are the important costimulatory molecules on DCs. These two markers engage in the interaction between APCs and T cells, which are key factors for the activation of APCs and immune responses[12]. CD83 is the typical mature marker of DCs. Although some of them did not reach statistical significance, the expression of CD80, CD83 and CD86 on imIFN-DC and imIL-4-DC was upregulated after the stimulation of CMV-DRibbles. Moreover, except for CD86, there is no different in the expression of HLA-DR, CD11c, CD80 and CD83 between imIFN-DC and imIL-4-DC after culture with CMV-DRibbles. Therefore, DCs loaded with DRibbles might be able to activate $\mathrm{T}$ cells through the increased expressions of costimulatory molecules and maturity.

To determine whether DRibbles could stimulate the cytokines secretion of moDCs, we compared the secretion of IL-6, IL-1 $\beta$, and IL-10 by mature moDCs and mature moDCs loaded with CMV-DRibbles. Although IFN-DC and IL-4-DC secreted IL-6 and 
IL-1 $\beta$ increased after TNF- $\alpha$ stimulation, CMVDRibbles did not further augment IL-6 or IL-1 $\beta$ production by DCs. Previous studies have demonstrated that differentiation of human Th17 cells from naïve $C D 4^{+} T$ cells requires IL- 6 and IL-1 $\beta$, and the effector function of memory Th17 cells needs IL-1 $\beta[25,26]$. Thus, we speculated that CMV-DRibbles had no ability to promote Th17 responses in $\mathrm{CD}^{+}$ $\mathrm{T}$ cells by DCs. As one of the important immune suppressive cytokines, IL-10 plays crucial roles in immune responses and inflammatory processes[27]. Not only the cells of the adaptive immune system, including $\mathrm{CD}^{+} \mathrm{T}$ cells, $\mathrm{CD} 8^{+} \mathrm{T}$ cells and $\mathrm{B}$ cells, could express IL-10 but also the cells from the innate immune system, such as DCs, natural killer cells, eosinophils, and neutrophils[27]. Here, in agreement with the literature, we found that both immature and mature IFN-DC produced plenty of IL-10[28]. However, CMV-DRibbles could not stimulate mIFN-DC to secrete more IL-10. In contrast, IL-4-DC did not express IL-10 whether with or without CMV-DRibbles stimulation. Although IFN-DCs produce low amounts of anti-inflammatory cytokines IL-10, IFN-DCs express numerous proinflammatory compared to IL-4-DC, making it unlikely to balance the excess amount of proinflammatory cytokines[5]. The above results were in contrast with a previous report that DRibbles could stimulate PBMCs to secrete cytokines [29]. On the account of the complexity of PBMCs, we did not know the exact cells types secreting cytokines under the stimulation of DRibbles, which needs future study. Taken together, our data suggested that CMV-DRibbles had no ability to stimulate cytokines secretion by moDCs.
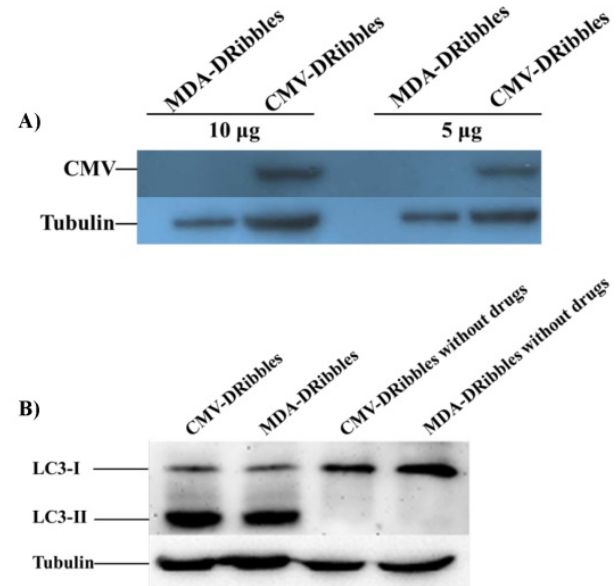

Figure 4. Western blot analysis of tumor cell derived DRibbles. MDA cells with or without the expression of CMV-pp65 protein were treated with 10 $\mathrm{mmol} / \mathrm{L} \mathrm{NH}{ }_{4} \mathrm{CL}$ and $100 \mathrm{nmol} / \mathrm{L}$ Bortezomib for 48 hours, respectively. The untreated MDA cells were as control. DRibbles were prepared from each group. (A) The expression of CMV-pp65 protein in CMV-DRibbles or MDA-DRibbles were detected by western blot analysis. (B) Autophagosome marker LC3 detected by Western blot analysis.
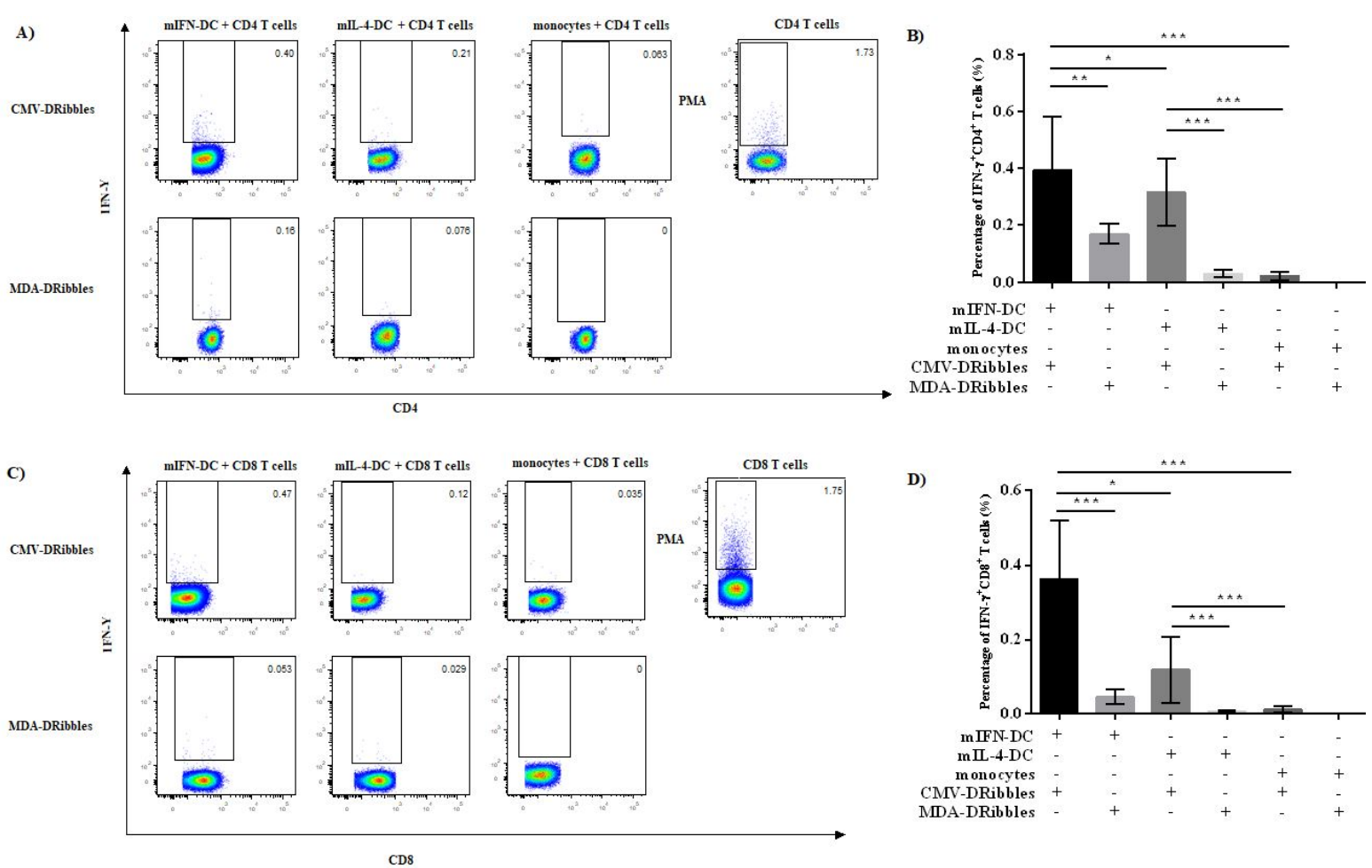

Figure 5. The activation of CMV antigen-specific CD4+ and CD8+ $T$ cells after stimulation of mIFN-DC and mIL-4-DC loaded with CMV-DRibbles. the mIFN-DC or mIL-4-DC was loaded with CMV-DRibbles or MDA-DRibbles for 12 hours. Monocytes were used as negative control. Then, DCs loaded with DRibbles were washed twice in PBS, and autologous T cells were added and cultured for another 12 hours. CD4 $4^{+}$or CD8 $8^{+}$T cells stimulated by PMA were used as positive control. The percentage of IFN- $\gamma^{+} \mathrm{CD} 4+\mathrm{T}$ cells $(\mathrm{A}, \mathrm{B})$ and IFN- $\mathrm{\gamma}^{+} \mathrm{CD} 8^{+} \mathrm{T}$ cells $(\mathrm{C}, \mathrm{D})$ were detected by intracellular staining. Results were represented as means $\pm \mathrm{SEMs}$ obtained from 5 independent experiments from five different donors. Statistical analysis was performed with One-way ANOVA followed by post-hoc test $(* p<0.05$, $* * *<0.01$, $* * * p<0.001)$. 
The cross-presentation efficiency of moDCs is not solely determined by the types of antigens; it is also affected by antigen uptake. To determine the uptake of CMV-DRibbles by these two moDCs, we compared the uptake of CMV-DRibbles by IFN-DC and IL-4-DC under different maturity of DCs, concentrations of CMV-DRibbles, and timepoints. Out of our expectation, no matter for IFN-DC or IL-4-DC, the uptake of CMV-DRibbles by mature moDCs was superior than immature moDCs, which was in contrast with some literature[30, 31]. We speculated that DRibbles containing multiple antigens might enhance the ability of uptake by mature DCs. Excellent antigen phagocytosis is beneficial to antigen presentation. Therefore, this result also proved that DRibbles were superior tumor antigens for DCs vaccines. Next, we observed that the uptake of CMV-DRibbles by mature DCs occurred in a dose-dependent manner. The dose of $50 \mu \mathrm{g} / \mathrm{mL}$ for DRibbles was enough for both IFN-DC and IL-4-DC. Then, we found that the best timepoint for the uptake of antigens by mIFN-DC was at 12 hours after loading with DRibbles, while the best timepoint for mIL-4-DC was at 24 hours. A previous study proves that these two moDCs have different antigen transport paths[32]. The antigen that enters DCs interacts with MHC-I. IFN-DC MHC-I molecules preferentially reside in the early endosomes, which is beneficial for cross-presentation, while IL-4-DC MHC-I molecules reside in traditional ER-Golgi compartment. This could explain the different efficiency of DRibbles uptake between IFN-DC and IL-4-DC.

At last, to determine whether DCs could be efficient in presenting or cross-presenting DRibbles to $\mathrm{T}$ cells, we compared the function of presenting CMV-DRibbles between IFN-DC and IL-4-DC by detecting IFN- $\gamma$ secretion by CMV specific T cells. We chose CMV-DRibbles derived from MDA cell lines expressing the CMV pp65 protein as the antigen because pp65 was a dominant antigen recognized by both CMV-specific $\mathrm{CD}^{+}{ }^{+}$and $\mathrm{CD}^{+} \mathrm{T}$ cells in humans with a history of CMV infection[33]. As expected, both IFN-DC and IL-4-DC could present CMV-DRibbles and activate CMV-specific $\mathrm{T}$ cells. These results indicated that tumor cell-derived DRibbles serving as a source of antigens were superior in activating antigen-specific $\mathrm{T}$ cells when loaded onto moDCs. This function might lead to suppression of tumor growth, which has been proven in a mouse model[12]. Furthermore, we observed that IFN-DC had a more efficient ability of presenting and cross-presenting CMV-DRibbles to $\mathrm{CD}^{+}$and $\mathrm{CD} 8^{+} \mathrm{T}$ cells compared with IL-4-DC. This observation is consistent with a study by Mohamad et al. also showing that IFN-DC was more efficient than IL-4-DC to induce antigen-specific immune response[22]. To data, no study has systematically reported the reasons for the difference in efficiency of presentation between these two moDCs. According to the report, IFN-DC expresses a broad spectrum of Toll-like receptors (TLRs) compared with IL-4-DC and TLRs play an essential role in the activation of innate immunity [22]. Meanwhile, a previous study proved that DRibbles contain many endogenous agonists for multiple TLRs[29]. Thus, based on the above results and previous reports, we speculated that the difference in TLRs expression might cause the different presentation efficiencies between these two moDCs, which is being investigated in our lab currently.

In summary, we found that DRibbles influenced the phenotype of moDCs. In addition, DRibbles had no ability to stimulate moDCs to secrete cytokines. Furthermore, we optimized the uptake condition of DRibbles by IFN-DC and IL-4-DC. At last, we detected that IFN-DC had superior ability in the aspect of presenting and cross-presenting DRibbles compared with IL-4-DC. Our study confirmed the ability of DRibbles to activate antigen-specific CD4 ${ }^{+}$ and $\mathrm{CD}^{+} \mathrm{T}$ cells in humans, suggesting that DRibbles might be an excellent antigen for priming anti-cancer immune responses in patients. A phase I clinical trial has proved that the autologous DRibbles vaccine is found to be safe when combined with docetaxel plus GM-CSF[34]. Therefore, the development of tumor vaccines based on IFN-DC and DRibbles is very valuable for the future.

\section{Abbreviations}

APC: antigen presenting cells; CMV: cytomegalovirus; DCs: Dendritic cells; DRibbles: defective ribosomal products in blebs; FACS: fluorescence activated cell sorting; FBS: fetal bovine serum; GM-CSF: granulocyte macrophage-colony stimulating factor; IFN-a: Interferon- $\alpha$; IL-4: interleukin (IL)-4; mAbs,: monoclonal antibodies; MHC: major histocompatibility complex; moDCs: monocytederived DCs; PBMC: peripheral blood mononuclear cells; TAA: tumor-associated antigens; Th17: T helper cell 17; TNF-a: tumor necrosis factor (TNF)- $\mathrm{a}$.

\section{Acknowledgements}

We thank Dr. Hong-ming Wu from Robert W. Franz Cancer Research Cente in USA for the generous gift of MDA human breast cancer cells and MDA human breast cancer cells expressing the CMV pp65 protein. This research was partially supported by grants from Nanjing Medical Science and technology development Foundation (No.YKK17167 to Jing Fan and No.YKK17173 to Wei Ye), the National Natural 
Science Foundation of China (No.81402559 to Wei Ye), the Science and Technology Commission of Nanjing (No.201605033 to Wei Ye), the Project of Jiangsu Provincial Medical Youth Talent (Wei Ye), the Project of Six Talent Peaks of Jiangsu Province (No.WSN-177 to Wei Ye) and the Jiangsu Provincial Special Program of Medical Science (NO.BL2014005 to Yongxiang Yi).

\section{Competing Interests}

The authors have declared that no competing interest exists.

\section{References}

1. Jongbloed SL, Kassianos AJ, McDonald KJ, Clark GJ, Ju X, Angel CE, et al. Human CD141+ (BDCA-3)+ dendritic cells (DCs) represent a unique myeloid DC subset that cross-presents necrotic cell antigens. J Exp Med. 2010; 207: 1247-60.

2. Anguille S, Smits EL, Lion E, van Tendeloo VF, Berneman ZN. Clinical use of dendritic cells for cancer therapy. The Lancet Oncology. 2014; 15: e257-e67.

3. Podrazil M, Horvath R, Becht E, Rozkova D, Bilkova P, Sochorova K, et al. Phase I/II clinical trial of dendritic-cell based immunotherapy (DCVAC/PCa) combined with chemotherapy in patients with metastatic, castration-resistant prostate cancer. Oncotarget. 2015; 6: 18192-205.

4. Dillman RO, Cornforth AN, Depriest C, McClay EF, Amatruda TT, de Leon C, et al. Tumor stem cell antigens as consolidative active specific immunotherapy: a randomized phase II trial of dendritic cells versus tumor cells in patients with metastatic melanoma. Journal of immunotherapy (Hagerstown, Md : 1997). 2012; 35: 641-9.

5. Farkas Á, Kemény L. Interferon- $\alpha$ in the generation of monocyte-derived dendritic cells: recent advances and implications for dermatology. British Journal of Dermatology. 2011; 165: 247-54.

6. Lin N-Y, Beyer C, Gießl A, Kireva T, Scholtysek C, Uderhardt S, et al. Autophagy regulates TNFa-mediated joint destruction in experimental arthritis. Annals of the Rheumatic Diseases. 2013; 72: 761

7. Cunha LD, Martinez J. Chapter 16 - Autophagy and LC3-Associated Phagocytosis Mediate the Innate Immune Response A2 - Hayat, M.A. Autophagy: Cancer, Other Pathologies, Inflammation, Immunity, Infection, and Aging: Academic Press; 2017. p. 303-19.

8. Shibutani ST, Saitoh T, Nowag H, Munz C, Yoshimori T. Autophagy and autophagy-related proteins in the immune system. Nature immunology. 2015; 16: 1014-24

9. Münz C. Autophagy proteins in antigen processing for presentation on MHC molecules. Immunological Reviews. 2016; 272: 17-27.

10. Yi Y, Zhou Z, Shu S, Fang Y, Twitty C, Hilton TL, et al. Autophagy-assisted antigen cross-presentation. OncoImmunology. 2012; 1: 976-8.

11. Li Y, Wang L-X, Yang G, Hao F, Urba WJ, Hu H-M. Efficient Cross-presentation Depends on Autophagy in Tumor Cells. Cancer Research. 2008; 68: 6889

12. Su H, Luo Q, Xie H, Huang X, Ni Y, Mou Y, et al. Therapeutic antitumor efficacy of tumor-derived autophagosome (DRibble) vaccine on head and neck cancer. International Journal of Nanomedicine. 2015; 10: 1921-30.

13. Ye W, Xing Y, Paustian C, van de Ven R, Moudgil T, Hilton TL, et al. Cross-presentation of viral antigens in dribbles leads to efficient activation of virus-specific human memory T cells. J Transl Med. 2014; 12: 100.

14. Klionsky DJ, Cuervo AM, Seglen PO. Methods for Monitoring Autophagy from Yeast to Human. Autophagy. 2007; 3: 181-206.

15. Miao P-Z, Cheng J, Okrah K, Liu B, Sun C, Cao G, et al. Abstract 1750: Analysis of non-metastatic HCC patient tumors revealed the significance of cell cycle regulation and tumor immunity in association with overall survival and identified clinically relevant druggable targets. Cancer Research. 2017; 77: 1750.

16. Van Woensel M, Mathivet T, Wauthoz N, Rosiere R, Garg AD. Sensitization of glioblastoma tumor micro-environment to chemo- and immunotherapy by Galectin-1 intranasal knock-down strategy. 2017; 7: 1217.

17. Nicolini A, Barak V, Biava P, Ferrari P, Rossi G, Carpi A. Use of immunotherapy to treat metastatic breast cancer. Current medicinal chemistry. 2018

18. Palucka K, Banchereau J. Dendritic-Cell-Based Therapeutic Cancer Vaccines. Immunity. 2013; 39: 38-48.

19. Blum JS, Wearsch PA, Cresswell P. Pathways of antigen processing. Annu Rev Immunol. 2013; 31: 443-73.

20. Mintern JD, Macri C, Chin WJ, Panozza SE, Segura E, Patterson NL, et al. Differential use of autophagy by primary dendritic cells specialized in cross-presentation. Autophagy. 2015; 11: 906-17.

21. Li Y, Wang L-X, Pang P, Cui Z, Aung S, Haley D, et al. Tumor-Derived Autophagosome Vaccine: Mechanism of Cross-Presentation and Therapeutic Efficacy Clinical Cancer Research. 2011; 17: 7047.
22. Mohty M, Vialle-Castellano A, Nunes JA, Isnardon D, Olive D, Gaugler B. IFN-a Skews Monocyte Differentiation into Toll-Like Receptor 7-Expressing Dendritic Cells with Potent Functional Activities. The Journal of Immunology. 2003; 171: 3385.

23. Bottazzo G, Hanafusa T, Pujol-Borrell R, Feldmann M. ROLE OF ABERRANT HLA-DR EXPRESSION AND ANTIGEN PRESENTATION IN INDUCTION OF ENDOCRINE AUTOIMMUNITY. The Lancet. 1983; 322: 1115-9.

24. Sadhu C, Ting HJ, Lipsky B, Hensley K, Garcia-Martinez LF, Simon SI, et al. CD11c/CD18: novel ligands and a role in delayed-type hypersensitivity. Journal of Leukocyte Biology. 2007; 81: 1395-403.

25. Acosta-Rodriguez EV, Napolitani G, Lanzavecchia A, Sallusto F. Interleukins 1 beta and 6 but not transforming growth factor-beta are essential for the differentiation of interleukin 17-producing human $\mathrm{T}$ helper cells. Nature immunology. 2007; 8: 942-9.

26. Wilson NJ, Boniface K, Chan JR, McKenzie BS, Blumenschein WM, Mattson JD, et al. Development, cytokine profile and function of human interleukin 17-producing helper T cells. Nature immunology. 2007; 8: 950-7.

27. Saraiva M, O'Garra A. The regulation of IL-10 production by immune cells. Nature reviews Immunology. 2010; 10: 170-81.

28. Caterina L, et al. IFN- $\alpha$-conditioned dendritic cells are highly efficient in inducing cross-priming CD8+ $\mathrm{T}$ cells against exogenous viral antigens. European Journal of Immunology. 2006; 36: 2046-60.

29. Xing Y, Cao R, Hu HM. TLR and NLRP3 inflammasome-dependent innate immune responses to tumor-derived autophagosomes (DRibbles). Cell death \& disease. 2016; 7: e2322.

30. Wertel I, Barczynski B, Kotarski J. The role of dendritic cells in cytotoxic immune response regulation in ovarian cancer micro-environment. Front Biosci; 2008. p. 2177-90

31. Hilkens CMU, Isaacs JD, Thomson AW. Development of Dendritic Cell-Based Immunotherapy for Autoimmunity. International Reviews of Immunology. 2010; 29: 156-83.

32. Spadaro F, Lapenta C, Donati S, Abalsamo L, Barnaba V, Belardelli F, et al. IFN- $\alpha$ enhances cross-presentation in human dendritic cells by modulating antigen survival, endocytic routing, and processing. Blood. 2012; 119: 1407.

33. Lore K, Betts MR, Brenchley JM, Kuruppu J, Khojasteh S, Perfetto S, et al. Toll-like receptor ligands modulate dendritic cells to augment cytomegalovirus- and HIV-1-specific T cell responses. Journal of immunology (Baltimore, Md : 1950). 2003; 171: 4320-8.

34. Sanborn RE, Ross HJ, Aung S, Kurup A, Moudgil T, Puri S, et al. A pilot single institution study of autologous tumor autophagosome (DRibble) vaccination with docetaxel in patients (pts) with stage IV non-small cell lung cancer (NSCLC). 2013. p. 30. 